\title{
Dihydroartemisinin increases radiosensitivity of A549 lung cancer cells
}

\author{
J. Li' ${ }^{1}$, X.Y. Li², S.L. Wu ${ }^{3}$, K. Liao ${ }^{*}$ \\ ${ }^{1}$ Department of Pharmacy, The Fifth People's Hospital of Chongqing, Chongqing, China \\ ${ }^{2}$ Department of Radiology, The First Affiliated Hospital of Chongqing Medical University, Chongqing, China \\ ${ }^{3}$ Department of Radiotherapy Center, The First Affiliated Hospital of Chongqing Medical University, Chongqing, \\ China
}

\begin{abstract}
- Original article

*Corresponding authors:

Dr. Kui Liao,

E-mail: 1187612268@qq.com

Revised: November 2019

Accepted: January 2020

Int. J. Radiat. Res., October 2020; 18(4): 707-713

DOI: 10.18869 /acadpub.ijrr.18.4.707

Background: Radiotherapy is the gold standard in the treatment of lung cancer. However, the radiosensitization of cancerous cells requires further improvement. Here, we investigated the effect of dihydroartemisinin (DHA) on the radiosensitization of non-small cell lung cancer (NSCLC) cells. Methods: Cell proliferation and cell cycle assays were carried out using A549 cells exposed to DHA. The effect of DHA on the radiosensitization of cells was investigated by clonogenic cell survival assay and apoptosis assay. Polymerase chain reaction and western blotting were used to quantify GSK-3 $\beta$ level. Results: DHA significantly inhibited cell proliferation from 24 to $96 \mathrm{~h}$. Cells treated with DHA had a significantly decrease G1 phase but significantly increase $S$ and $G 2 / M$ phases compared with untreated and vehicle-treated cells. The number of viable DHA-treated cell colonies was significantly lower than that of untreated or vehicle-treated cells. The percentage of early apoptotic and necrotic cells was significantly higher for cells exposed to DHA and irradiation. GSK-3 $\beta$ expression was significantly higher in cells exposed to DHA and irradiation than that in untreated cells, indicating that DHA may enhances the radiosensitization of cells through the activation of GSK-3 3 . Conclusions: Our results demonstrate that DHA can increase the radiosensitivity of $A 549$ cells, suggested its potential use to sensitize tumors to radiation therapy in NSCLC. The expressions of GSK-3 $\beta$ were induced by DHA in cells exposed to irradiation, indicating GSK-3 $\beta$ may paly important role in the radiosensitization mechanism of DHA.
\end{abstract}

Keywords: Dihydroartemisinin, Non-small cell lung cancer, A549 cells, Radiosensitization, Glycogen synthase kinase-3beta.

\section{INTRODUCTION}

Lung cancer is one of the most common types of cancer worldwide, with an estimated 2.1 million new diagnoses and 1.8 million deaths each year (1). Among the different types of lung cancer, non-small cell lung cancer (NSCLC) constitutes approximately $85 \%$ of all lung cancers (2). Although new treatments, such as immunotherapy and targeted therapy, have been shown to improve the prognostic outcome, radiotherapy remains the gold standard in the treatment of early stage and advanced NSCLC (35). However, improvements are needed to enhance the radiosensitization of cancerous cells.

Artemisinin is a sesquiterpene lactone derived from Artemisia annua, a traditional Chinese medicinal plant (6). Dihydroartemisinin (DHA), an active metabolite of artemisinin, is an antimalarial drug that has recently been shown to exhibit potent anti-cancer effects (7-9). For instance, DHA can induce A549 cell apoptosis (10). We previously demonstrated that DHA can inhibit the proliferation of cells, and resulted in cell cycle arrest in NSCLC (11). Other studies have reported that artemisinin, artesunate, and DHA are toxic to radiation-resistant human breast 
cancer cells and drug-resistant human NSCLC cells, particularly when the cells are pretreated with transferrin, which increases the intracellular $\mathrm{Fe}^{+}$level $(12,13)$. Here, we hypothesized that DHA may improve the radiosensitivity of A549 cells. Glycogen Synthase Kinase-3 beta (GSK-3 $\beta$ ), a serine/threonine protein kinase, regulates radiosensitivity of tumors in several types of cancers $(14,15)$.

Thus, we hypothesized that DHA may enhance the radiosensitization of A549 cells through GSK-3 $\beta$. In this study, we explored the effect of DHA on the radiosensitization of A549 cells and assess the mechanism of action, aiming to provide ample evidence for potential use of DHA in sensitizing NSCLC to radiation therapy.

\section{MATERIALS AND METHODS}

\section{Cell and culture}

The non-small cell lung cancer cell line A549 was obtained from the Cell Bank of the Committee on the Type Culture Collection of the Chinese Academic of Science (CCTCC, Shanghai, China). Cells were seeded in flasks and cultured in Dulbecco's modified Eagle medium (DMEM) supplemented with $10 \%$ fetal bovine serum (FBS, Gibco), $100 \mathrm{U} / \mathrm{ml}$ penicillin, and $100 \mathrm{mg} /$ $\mathrm{ml}$ streptomycin in a humidified atmosphere of $5 \% \mathrm{CO}_{2}$ at $37^{\circ} \mathrm{C}$. Cells were exposed to radiation, which was generated with a linear accelerator (UNIQUE; Varian, Palo Alto, CA, USA).

\section{Determination of the IC10 of DHA}

A549 cells were seeded in 96-well plates at $5.0^{\prime} 10^{4} /$ well, incubated for $9 \mathrm{~h}$, and then exposed to different concentrations of DHA for three days. Cells of the blank group were treated with DMEM, and those of the control group were treated with DMEM containing 1\% dimethyl sulfoxide (vehicle). The absorbance at wavelength $450 \mathrm{~mm}$ was measured after three days.

\section{Cell proliferation assay}

Cell proliferation was assessed with the Cell Count Kit-8 (CCK-8 Beyotime, China), according to the manufacturer's instructions. A549 cells were seeded in 96-well plates at $5.0^{\prime} 10^{4}$ /well and incubated for four days. Cells of the blank group were treated with DMEM, and those of the control group were treated with DMEM containing 1\% DMSO. The absorbance at wavelength $450 \mathrm{~mm}$ was measured from days 1 to 4 .

\section{Cell cycle assay}

The cell cycle was analyzed by flow cytometry (FCM) with propidium iodide staining. Both attached and detached cells were collected by trypsin digestion, centrifuged at low speed, washed with ice-cold phosphate buffered saline (PBS), and then fixed in ice-cold $70 \%$ ethanol overnight. Cells were collected by a brief centrifugation step and resuspended in PBS, after which they were treated with RNase A, stained with propidium iodide for $1 \mathrm{~h}$ at room temperature, and analyzed by FCM.

\section{Clonogenic cell survival assay}

A549 cells were seeded in 6-well plates at $200^{\prime} 10^{4} /$ well and cultured in the presence or absence of DHA (DMEM or vehicle) for $6 \mathrm{~h}$. Thereafter, DHA-treated and untreated cells were exposed to 2-Gy irradiation. Cells were incubated for 14 days, fixed with ethanol, and then stained with $0.1 \%$ crystal violet. Colonies with more than 50 cells were counted under a Leica DM4B microscope (Wetzlar, Germany).

\section{Apoptosis assay}

A549 cells were seeded in 6-well plates at $5^{\prime}$ $10^{5} /$ well and cultured in the presence or absence (DMEM or vehicle) of DHA for $6 \mathrm{~h}$. Thereafter, DHA-treated and untreated cells were exposed to 2-Gy irradiation. Cells were harvested and washed twice with PBS. Cell viability was assessed with the Annexin V-PE Apoptosis Kit (BD Biosciences, Franklin Lakes, NJ, USA), according to the manufacturer's instructions. The samples were immediately analyzed by FCM.

\section{Quantitative PT-PCR}

Total RNA was reverse transcribed into cDNA with the One-Step SYBR PrimeScript RT-PCR Kit 
II (Takara Biotechnology, Dalian, China), and qRT-PCR was performed using SYBR Premix Ex Taq (Takara Biotechnology) according to the manufacturer's instructions. The primers for GSK-3 $\beta$ were $5^{\prime}$-ACTGTGTAGCCGTCTGCTGGAG$3^{\prime}$ (forward) and $5^{\prime}-$ CAGGTGGAGTTGGAAGCTGATGC-3' (reverse). The primers for GAPDH were $5^{\prime}-$ ACCTGACCTGCCGTCTAGAA-3' (forward) and $5^{\prime}$ -TCCACCACCCTGTTGCTGTA-3' (reverse). The results were analyzed by the ${ }^{\Delta \Delta} \mathrm{Ct}$ method. Each experiment was repeated three times.

\section{Antibodies and western blotting}

The antibodies used in this study were GSK-3 $\beta$ (Cell Signaling Technology, Danvers, MA, USA) and $\beta$-ACTIN (Santa Cruz Biotechnology, Santa Cruz, CA, USA). Proteins were extracted from cells with RIPA buffer (Beyotime Institute of Biotechnology, Nantong, China), separated on a sodium dodecyl sulfate polyacrylamide gel, and transferred to a polyvinylidene fluoride membrane (Millipore, Billerica, MA, USA). Antibody--antigen complexes were detected using enhanced chemiluminescence reagents (Advansta, Menlo Park, CA, USA). The blots were scanned with the ChemiDoc Touch Imaging System (Bio-Rad, Hercules, CA, USA) and analyzed with the Image Lab Software (Bio-Rad, Hercules, CA, USA).

\section{Statistical analysis}

All data were analyzed using SPSS 21.0 Software (SPSS Inc., Chicago, IL, USA). Statistical data are presented as the mean \pm standard deviation (SD). Comparisons of continuous variables between two groups were performed using Student's $t$-test. Univariate analysis of variance was used for comparisons among three or more groups. $P$-values less than 0.05 were considered statistically significant.

\section{RESULTS}

\section{Determination of the IC10 of DHA}

To determine the IC10 of DHA, A549 cells were treated with different concentrations DHA for three days, as shown in table 1 . The IC10 of DHA was $23.47 \mu \mathrm{M}$.

Table 1. The effect of different concentrations of DHA on A549 cells.

\begin{tabular}{|c|c|c|}
\hline $\begin{array}{c}\text { Concentration } \\
\left(\mu \mathrm{mol} \cdot \mathrm{L}^{-1}\right)\end{array}$ & $\mathrm{OD}_{450}$ & $\begin{array}{c}\text { Inhibitory rate } \\
(\%)\end{array}$ \\
\hline 300 & $0.19 \pm 0.06$ & 100.00 \\
\hline 250 & $0.27 \pm 0.13$ & 92.17 \\
\hline 200 & $0.38 \pm 0.09$ & 83.15 \\
\hline 150 & $0.45 \pm 0.15$ & 70.67 \\
\hline 100 & $0.56 \pm 0.10$ & 58.96 \\
\hline 75 & $0.64 \pm 0.14$ & 47.13 \\
\hline 60 & $0.69 \pm 0.12$ & 36.24 \\
\hline 45 & $0.72 \pm 0.13$ & 23.36 \\
\hline 30 & $0.78 \pm 0.15$ & 15.87 \\
\hline 15 & $0.81 \pm 0.16$ & 9.16 \\
\hline
\end{tabular}

\section{DHA inhibits the proliferation of A549 cells}

A549 cells were treated with $23 \mu \mathrm{M}$ DHA for $96 \mathrm{~h}$, and the effect of DHA on the proliferation of cells was determined with the CCK-8 Kit. As shown in figure 1, DHA significantly inhibited cell proliferation from 24 to $96 \mathrm{~h}$ compared with untreated and vehicle-treated cells $(p<0.05)$.

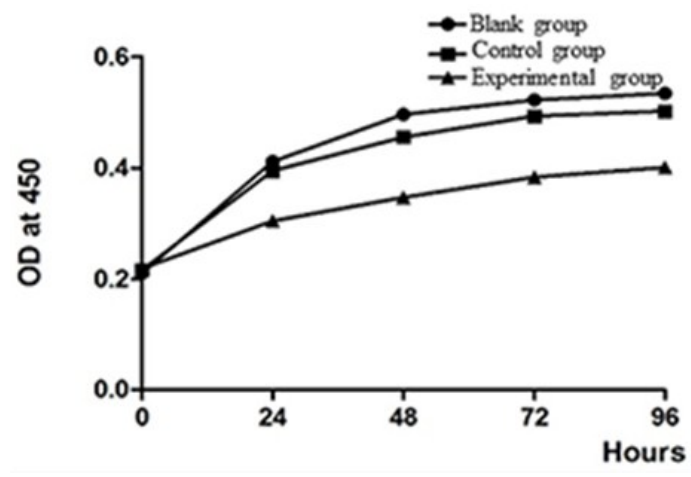

Figure 1. The influence of dihydroartemisinin on the proliferation of A549 cells was detected. DHA significantly inhibited $A 549$ cell proliferationrom from the 24th hour to the 96th hour.

\section{DHA induces cell cycle arrest in $A 549$ cells}

To examine the effect of DHA on the cell cycle, A549 cells were treated with $23 \mu \mathrm{M}$ of DHA for $48 \mathrm{~h}$. As shown in figure 2, cells treated with DHA had a significantly decrease G1 phase, but significantly increase $\mathrm{S}$ and $\mathrm{G} 2 / \mathrm{M}$ phases compared with untreated and vehicle-treated 
cells $(p<0.05)$. These findings indicate that DHA arrested A549 cells at the S phase and the G2/M phase.

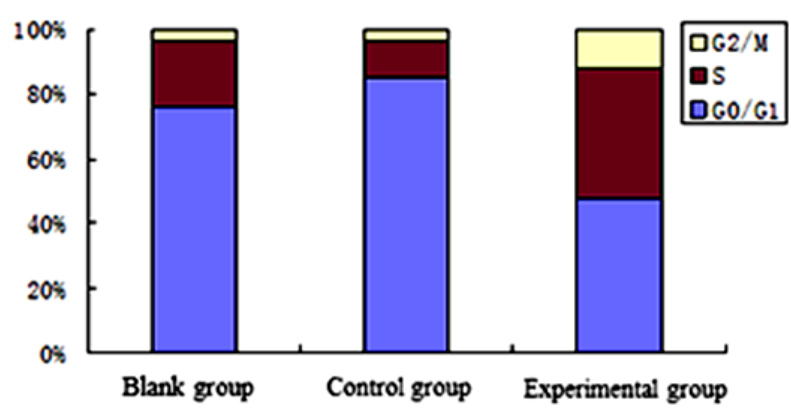

Figure 2. Dihydroartemisinin induced cell cycle arrest in A549 cells. Cells treated with DHA showed a decrease in G1 phase and an increase in both $S$ phase and $G 2 / M$ phase

\section{DHA enhances the radiosensitization of $A 549$ cells}

To examine the effect of DHA on the radiosensitization of $A 549$ cells, both clonogenic cell survival and apoptosis assays were performed. As shown in figure $3 \mathrm{~A}$, the number of viable DHA-treated cell colonies was significantly lower than that of untreated or vehicle-treated cells $(p<0.05)$. As shown in figure $3 \mathrm{~B}$, the percentage of early apoptotic and necrotic cells was significantly higher for cells exposed to DHA and irradiation than that for untreated or vehicle-treated cells $(p<0.05)$. These findings indicate that DHA increased the radiosensitivity of A549 cells.

\section{DHA enhances the radiosensitization of $A 549$ cells by activating GSK-3 $\beta$}

GSK-3 $\beta$ expression was examined in untreated A549 cells, cells exposed to irradiation, and cells exposed to both DHA and irradiation. As shown in Figure 4A, the GSK-3 $\beta$ mRNA level was significantly higher in cells exposed to both DHA and irradiation than that in untreated cells or those exposed to irradiation only $(p<0.05)$, consistent with the results of western blotting (figure 4B). These findings speculate DHA enhanced the radiosensitization of A529 cells through GSK-3 $\beta$ activation.

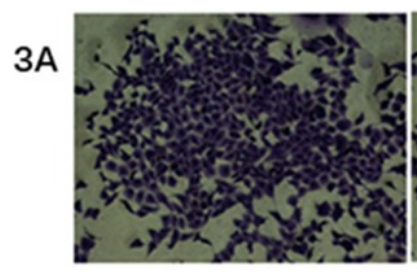

Blank group

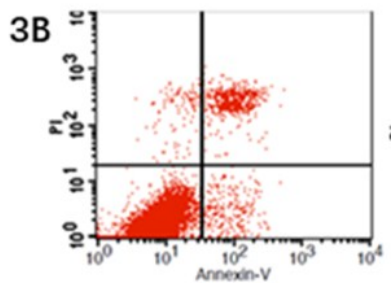

Blank group

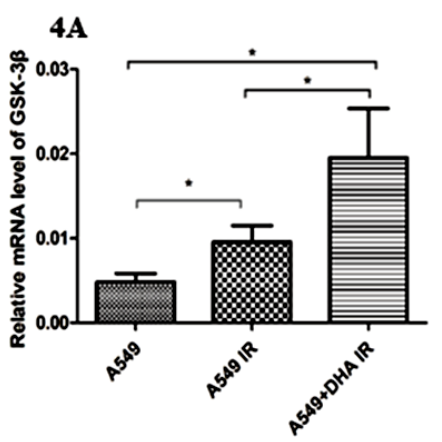

710

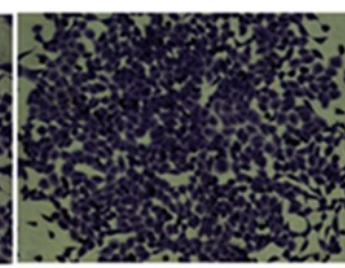

Control group

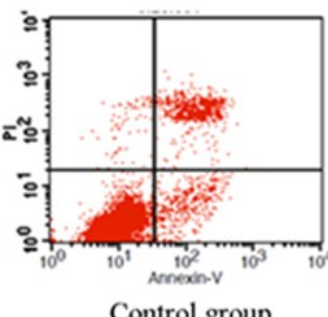

$4 B$

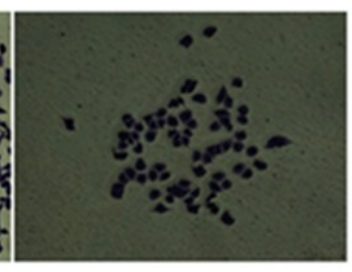

Experimental group

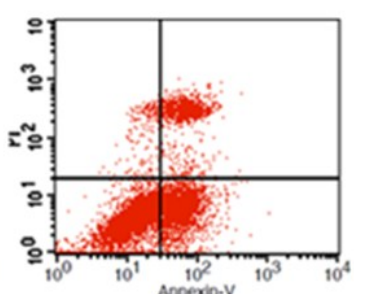

Experimental group
Figure 3. In clone formation experiment, dihydroartemisinin increased $\mathrm{A} 549$ radiation sensitivity (magnification in 100) (3A).

Dihydroartemisinin increased the apoptosis of A549 cells after radiotherapy (3B).
Figure 4. The expression of GSK3 $\beta$ was significantly higher in the experimental group (A549 cells treated with both DHA and irritation) among the three groups in both mRNA level $(4 A)$ and protein level $(4 B)(p<0.05)$. 


\section{DISCUSSION}

Artemisinin and its derivatives (i.e., DHA) have been one of the drugs subject to drug repurposing with promising benefits in the field of oncology. The artemisinin-type drugs have been closely combined with various cancer therapies, such as standard chemotherapy, radiotherapy and photodynamic therapy (16). Artemesinin has been tested as a radiosensitizer in glioblastoma cells (17), and Hela cell lines (18), and A549 lung cancer cells (19). DHA has been studied as a radiosensitizer in glioblastoma (20), and cervical cancer(21), and lung cancer cell lines(22). These studies have shown the radiosensitizing effects of artemisinin and dihydroartemesinin, with induction of apoptosis and G2/M cell cycle arrest. The current study has confirmed these findings in addition it has shown $\mathrm{S}$ phase accumulation and growth arrest. Such findings suggest that dihydroartemisinin may be a potential radiotherapy sensitization in non-small cell lung cancer.

Furthermore, to examine the underlying radiosensitization mechanism of DHA in A549 cells, we analyzed the expression of GSK-3 $\beta$. GSK $-3 \beta$, a multifunctional serine/threonine kinase, had been initially identified as a key regulator of insulin-dependent glycogen synthesis. GSK-3 $\beta$ interaction with various proteins; for example, GSK-3 $\beta$ stabilizes or activates mouse double minute 2 homolog (MDM2) and destabilizes or inactivates activator protein 1 (AP-1)(23). Also, GSK-3 $\beta$ is activated by various pathways, including the PI3K/Akt and Wnt pathways (24-26). Therefore, GSK-3 $\beta$ regulates a diverse array of cellular processes including proliferation, differentiation, motility and survival (27). Consequently, the role of GSK3 $\beta$ in tumorigenesis and cancer progression remains controversial. It may function as a tumor suppressor for certain types of tumors $(27,28)$, but promotes growth and development for some others (29-31).

Although, previous studies have shown that GSK-3 $\beta$ expression is associated with NSCLC differentiation, and patients with GSK-3 $\beta$ negative tumors had a better prognosis $(31,32)$; on the other hand, studies have also demonstrated that GSK-3 $\beta$ inhibits autophagy $(33,34)$, which facilitates the removal of unwanted mitochondria, thereby decreasing the radiosensitivity of cancerous cells and protecting them from the effects of irradiation (35). Ren J et $a l$. has proved that GSK-3 $\beta$ can enhance the radiosensitivity of NSCLC cells, both in GSK-3 $\beta$ high A549 cell line and in GSK-3 $\beta$-low H460 cell line (32). In our study, we found that GSK-3 $\beta$ level was significantly higher in cells treated with DHA than that in untreated or vehicle-treated cells, indicating DHA induced GSK-3 $\beta$ expression and increased the radiosensitivity of cells.

However, more studies are still needed to explore the radiosensitization mechanism of DHA. On the other hand, Li X et al. have investigated the cytotoxicity of artemisinin and artesunate on A549 cell line and on human bronchial epithelium. They demonstrated a cytotoxic effect on both cell lines but higher in A549 cell line (36). Our current study only investigates the radiosensitization on lung cancer cell lines. Further studies are still needed to clear the cytotoxicity of DHA on non-cancer tissues with and without radiotherapy.

\section{CONCLUSION}

In conclusion, DHA can inhibit the proliferation of A549 cells and arrest cell cycle. DHA also increased the radiosensitivity of A549 cells, suggested its potential use to sensitize tumors to radiation therapy in NSCLC. In addition, DHA induced the expression of GSK-3 $\beta$ in cells exposed to irradiation, indicating GSK-3 $\beta$ may paly important role in the radiosensitization mechanism of DHA.

\section{Authors' contributions}

Kui Liao and Juan Li contributed to concept and design; Kui Liao provided administrative support; Juan Li provided study materials; Xinyou $\mathrm{Li}$ collected and assembled data; Shunlong $\mathrm{Wu}$ analyzed data; all authors wrote the manuscript and approved the final version of the manuscript. 


\section{Li, Wu, Liao / Dihydroartemisinin increased radiosensitivity in A549 cells}

\section{Funding}

This study was supported by the Science and Technology Project Foundation of Yuzhong District of Chongqing (Grant No. 20170125).

\section{Abbreviations \\ NSCLC non-small cell lung cancer \\ DHA dihydroartemisinin.}

\section{Conflicts of interest: Declared none.}

\section{REFERENCES}

1. Organization WH ( January 6, 2019) Cancer Fact Sheet, 2018.

2. Miller KD, Siegel RL, Lin CC (2016) Cancer treatment and survivorship statistics, 2016. CA: A Cancer Journal for Clinicians, 66(4): 271-89.

3. Chua KLM, Sin I, Fong KW, Chua MLK, Onishi H (2017) Stereotactic body radiotherapy for early stage lung cancerhistorical developments and future strategies. Chinese Clinical Oncology, 6(Suppl 2): S2O.

4. Auperin A, Le Pechoux C, Rolland E, Curran WJ, Furuse K, Fournel P, et al. (2010) Meta-analysis of concomitant versus sequential radiochemotherapy in locally advanced non -small-cell lung cancer. Journal of Clinical Oncology, 28 (13): 2181-90.

5. Cheema PK, Rothenstein J, Melosky B, Brade A, Hirsh V (2019) Perspectives on treatment advances for stage III locally advanced unresectable non-small-cell lung cancer. Current Oncology, 26(1): 37-42.

6. Hao X, Zhong Y, Fu X, Lv Z, Shen Q, Yan T, et al. (2017) Transcriptome Analysis of Genes Associated with the Artemisinin Biosynthesis by Jasmonic Acid Treatment under the Light in Artemisia annua. Frontiers in Plant Science, 8:971.

7. Li H, Li X, Shi X, Li Z, Sun Y (2018) Effects of magnetic dihydroartemisinin nano-liposome in inhibiting the proliferation of head and neck squamous cell carcinomas. Phytomedicine, 56: 215-28.

8. Liu Y, Gao S, Zhu J, Zheng Y, Zhang H, Sun H (2018) Dihydroartemisinin induces apoptosis and inhibits proliferation, migration, and invasion in epithelial ovarian cancer via inhibition of the hedgehog signaling pathway. Cancer Medicine, 7(11): 5704-15.

9. Wang D, Zhong B, Li Y, Liu X (2018) Dihydroartemisinin increases apoptosis of colon cancer cells through targeting Janus kinase 2/signal transducer and activator of transcription 3 signaling. Oncology Letters, 15(2): 1949-54.

10. Liu X, Wu J, Fan M, Shen C, Dai W, Bao Y, et al. (2018) Novel dihydroartemisinin derivative DHA-37 induces autophagic cell death through upregulation of HMGB1 in A549 cells. Cell Death \& Disease, 9(11): 1048.
11. Liao K, Li J, Wang Z (2014) Dihydroartemisinin inhibits cell proliferation via AKT/GSK3beta/cyclinD1 pathway and induces apoptosis in A549 lung cancer cells. Int J Clin Exp Path, 7(12): 8684-91.

12. Singh NP and Lai $H$ (2001) Selective toxicity of dihydroartemisinin and holotransferrin toward human breast cancer cells. Life Sciences, 70(1): 49-56.

13. Sadava D, Phillips T, Lin C, Kane SE (2002) Transferrin overcomes drug resistance to artemisinin in human small-cell lung carcinoma cells. Cancer Letters, 179(2): 151-6.

14. Miyashita K, Kawakami K, Nakada M, Mai W, Shakoori A, Fujisawa $\mathrm{H}$, et al. (2009) Potential therapeutic effect of glycogen synthase kinase $3 \beta$ inhibition against human glioblastoma. Clinical Cancer Research, 15(3): 887-97.

15. Kitano A, Shimasaki T, Chikano Y, Nakada M, Hirose M, Higashi T, et al. (2013) Aberrant glycogen synthase kinase 3beta is involved in pancreatic cancer cell invasion and resistance to therapy. PLoS One, 8(2): e55289.

16. Efferth T (2017) Cancer combination therapies with artemisinin-type drugs. Biochemical Pharmacology, 139: 5670.

17. Reichert S, Reinboldt V, Hehlgans S, Efferth T, Rodel C, Rodel F (2012) A radiosensitizing effect of artesunate in glioblastoma cells is associated with a diminished expression of the inhibitor of apoptosis protein survivin. Radiotherapy and Oncology, 103(3): 394-401.

18. Luo J, Zhu W, Tang Y, Cao H, Zhou Y, Ji R, et al. (2014) Artemisinin derivative artesunate induces radiosensitivity in cervical cancer cells in vitro and in vivo. Radiation Oncology, 9: 84 .

19. Zhao Y, Jiang W, Li B, Yao Q, Dong J, Cen Y, et al. (2011) Artesunate enhances radiosensitivity of human non-small cell lung cancer A549 cells via increasing NO production to induce cell cycle arrest at G2/M phase. International Immunopharmacology, 11(12): 2039-46.

20. Kim SJ, Kim MS, Lee JW, Lee $\mathrm{CH}, \mathrm{Yoo} \mathrm{H}$, Shin $\mathrm{SH}$, et al. (2006) Dihydroartemisinin enhances radiosensitivity of human glioma cells in-vitro. J Can Res Clin Oncol, 132(2): 129-35.

21. Luo J, Chen X, Chen G, Zhou X, Lu X, Ling Y, et al. (2013) Dihydroartemisinin induces radiosensitivity in cervical cancer cells by modulating cell cycle progression. Saudi Medical Journal, 34(3): 254-60.

22. Zuo ZJ, Wang ST, Jiang LX, Xin YX, Li W, Xu ZH, et al. (2014) Effect of dihydroartemisinin combined irradiation on the apoptosis of human lung cancer GLC-82 cells and its mechanism study. Zhongguo Zhong xi yi jie he za zhi Zhongguo Zhongxiyi jiehe zazhi = Chinese Journal of Integrated Traditional and Western Medicine, 34(10): 1220-4.

23. Domoto T, Pyko IV, Furuta T, Miyashita K, Uehara M, Shimasaki T, et al. (2016) Glycogen synthase kinase- $3 \beta$ is a pivotal mediator of cancer invasion and resistance to therapy. Cancer Science, 107(10): 1363-72.

24. Fang X, Yu SX, Lu Y, Bast RC, Jr., Woodgett JR, Mills GB (2000) Phosphorylation and inactivation of glycogen synthase kinase 3 by protein kinase A. Proceedings of the

Int. J. Radiat. Res., Vol. 18 No. 4, October 2020 
National Academy of Sciences of the United States of America, 97(22): 11960-5.

25. Wen SY, Chen YY, Deng CM, Zhang CQ, Jiang MM (2018) Nerigoside suppresses colorectal cancer cell growth and metastatic potential through inhibition of ERK/GSK3beta/ beta-catenin signaling pathway. Phytomedicine, 57: 35263.

26. Tejeda-Munoz N, Robles-Flores M (2015) Glycogen synthase kinase 3 in Wnt signaling pathway and cancer. IUBMB life, 67(12): 914-22.

27. Luo J (2009) Glycogen synthase kinase $3 \beta$ (GSK3 $\beta$ ) in tumorigenesis and cancer chemotherapy. Cancer Letters, 273(2): 194-200.

28. McCubrey JA, Davis NM, Abrams SL, Montalto G, Cervello M, Basecke J, et al. (2014) Diverse roles of GSK-3: tumor promoter-tumor suppressor, target in cancer therapy. Advances in Biological Regulation, 54: 176-96.

29. Kunnimalaiyaan $M$, Vaccaro AM, Ndiaye MA, Chen $H$ (2007) Inactivation of glycogen synthase kinase-3beta, a downstream target of the raf-1 pathway, is associated with growth suppression in medullary thyroid cancer cells. Molecular Cancer Therapeutics, 6(3): 1151-8.

30. Zhu Q, Yang J, Han S, Liu J, Holzbeierlein J, Thrasher JB, et al. (2011) Suppression of glycogen synthase kinase 3 activi- ty reduces tumor growth of prostate cancer in-vivo. Prostate, 71(8): 835-45.

31. Zeng J, Liu D, Qiu Z, Huang Y, Chen B, Wang L, et al. (2014) GSK3beta overexpression indicates poor prognosis and its inhibition reduces cell proliferation and survival of nonsmall cell lung cancer cells. PLoS One, 9(3): e91231.

32. Ren J, Liu T, Han Y, Wang Q, Chen Y, Li G, et al. (2018) GSK3beta inhibits autophagy and enhances radiosensitivity in non-small cell lung cancer. Diagnostic Pathology, 13(1): 33.

33. Yang $\mathrm{Y}$, Wang $\mathrm{H}$, Wang $\mathrm{S}$, Xu M, Liu $\mathrm{M}$, Liao $\mathrm{M}$, et al. (2012) GSK3beta signaling is involved in ultraviolet B-induced activation of autophagy in epidermal cells. International Journal of Oncology, 41(5): 1782-8.

34. Azoulay-Alfaguter I, Elya R, Avrahami L, Katz A, EldarFinkelman H (2015) Combined regulation of mTORC1 and lysosomal acidification by GSK-3 suppresses autophagy and contributes to cancer cell growth. Oncogene, 34(35): 4613-23.

35. Gewirtz DA (2014) Autophagy and senescence in cancer therapy. Journal of cellular physiology, 229(1): 6-9.

36. Li X, Gu S, Sun D, Dai H, Chen H, Zhang Z (2018) The selectivity of artemisinin-based drugs on human lung normal and cancer cells. Environmental Toxicology and Pharmacology, 57: 86-94. 
